University of San Diego

Digital USD

Spring 5-23-2015

\title{
Transition Program for Adolescents with Congenital Heart Disease Focusing on Self-Management Knowledge
}

\author{
Elizabeth A. Hartman Valles \\ University of San Diego, ehartmanvalles@sandiego.edu \\ Kathleen Sweeney CPNP \\ University of San Diego, ksweeney@sandiego.edu \\ Jeffrey Frazer M.D. \\ University of California San Diego, jfrazer@rchsd.org
}

Follow this and additional works at: https://digital.sandiego.edu/dnp

Part of the Health and Physical Education Commons, and the Medicine and Health Sciences Commons

\section{Digital USD Citation}

Hartman Valles, Elizabeth A.; Sweeney, Kathleen CPNP; and Frazer, Jeffrey M.D., "Transition Program for Adolescents with Congenital Heart Disease Focusing on Self-Management Knowledge" (2015). Doctor of Nursing Practice Final Manuscripts. 35.

https://digital.sandiego.edu/dnp/35

This Doctor of Nursing Practice Final Manuscript is brought to you for free and open access by the Theses and Dissertations at Digital USD. It has been accepted for inclusion in Doctor of Nursing Practice Final Manuscripts by an authorized administrator of Digital USD. For more information, please contact digital@sandiego.edu. 
Transition Program for Adolescents with Congenital Heart Disease Focusing on Self-

Management Knowledge

Elizabeth A. Hartman Valles DNP, PNP, FNP, CCRN, RCIS

Kathleen Sweeny DNP, CPNP

Jeffrey Frazer MD

Disclosures: none

Acknowledgements: Dr Moore, Dr. Perry

Key Words: transition, adolescent, congenital heart disease 


\begin{abstract}
Introduction: The primary goal of this project was to implement a pilot transition education program to prepare adolescents with congenital heart defects (CHD) by increasing knowledge about self-management of their health condition.

Method: An educational program was developed and included use of an interactive binder. A total of 20 adolescents with CHD between the ages of 16 - 18 years participated in transition education during a single outpatient cardiology visit with pre and post education knowledge assessment performed using the Leuven Knowledge Questionnaire for Congenital Heart Disease (LKQCHD).

Results: Following participation in the transition education, the overall mean knowledge scores increased from 43.6\% (+/-) to 89.4\%. (+/-). All participants scored $>50 \%$ post intervention.

Discussion: A formal transition education program demonstrated increased patient knowledge regarding their CHD. Advanced practice nurses are able to implement a transition education program and provide the necessary education to make a significant impact on patient knowledge.
\end{abstract}




\section{Transition Program for Adolescents with Congenital Heart Disease Focusing on Self-Management Knowledge}

Transitioning adolescents with complex medical conditions from pediatric to adult health care providers can be challenging, and many specialty clinics may lack the resources to provide formal transition programs. Adolescents with complex medical conditions are often unaware of the specifics related to their diagnosis and medical decisions are typically managed by their parents. Because of this, adolescents may not be prepared to enter the adult healthcare system and subsequent management of their complex medical condition. Blum et al. (1993, p. 570) defined transition as "the purposeful, planned movement of adolescents and young adults with chronic physical and medical conditions from child-centered to adult-oriented health care systems” (Anthony et al., 2009, p. 614). A formal Transition Program assists patients in fostering independence as they enter the adult health care environment.

Congenital heart defects (CHD) occur in approximately eight of 1000 births, with $90 \%$ of these patients now living to adulthood as a result of advancements in care (Anthony et al., 2009). In the absence of proper care, adolescents and adults with CHD could face serious mental and physical health consequences (Jalkut \& Allen, 2009). Less than 30\% of adults with CHD (ACHD) have health care providers who are trained in this specialty. (Sable et al., 2011).

Without a formalized program in place that can guide patients through transition, care can be delayed or inappropriate (Goossens, Van Deyk, Zupancic, Budts, \& Moons, 2014; Sable et al., 2011). This can result in patients being lost to follow-up. A wellplanned and executed transition for adolescents with CHD is important and should 
include developmentally appropriate education related to their medical conditions, and also foster competencies in communication, decision making, self-care and self-advocacy (Sable et al., 2011).

According to the 2009-2010 National Survey of Children with Special Health Care Needs (SHCN) only 37\% of eligible adolescents with SHCN in California met the criteria established for transition goals (McManus et al., 2013; U.S. Department of Health and Human Services, Health Resources and Services Administration, \& Bureau., 2013). Healthy People 2020 has set a goal that $45 \%$ of adolescents with SHCN participate in transition planning (Department of Health and Human Services, 2013). Current data supports minimal improvement in transition care since the benchmarks identified in Healthy People 2010.

\section{Description of Program Context}

Many factors contribute to adolescents with CHD not being prepared to selfmanage their condition as adults. Since most of these patients are diagnosed early in childhood, the caregiver usually assumes management. These patients are typically in a patient or family-centered care health care environment that may not require them to selfmanage their condition. Parents make the appointments and also facilitate diagnostic testing and follow-up appointments (Sable et al., 2011). Medication regimens and refills

are also managed by parents (Sable et al., 2011). Minimal levels of self management can result in a lack of knowledge by adolescents with CHD related to the name of their heart defect, symptoms of deterioration of their condition, and risk factors for endocarditis. In addition, they lack knowledge on how often they should have follow-up appointments, and if medications are required, they have limited knowledge on the purpose of the 
medication as well as the consequences of not taking it (Van Deyk et al., 2010). Lack of readiness of the parents to let go of the control of managing their child's condition, or the lack of readiness on the part of the patient to perform self-care activities related to their condition or self-manage their health care also contribute to the lack of transition (Sable et al., 2011).

Adolescents with SHCN have an increased risk for developmental problems, excessive dependency and psychosocial delay (Rosen, 2004). These factors can impede a successful transition to adult-oriented care. It is imperative that care be provided in a way that is "developmentally appropriate, improves capacity for self-reliance, increases a sense of personal responsibility and enhances autonomy and independence” (Rosen, 2004, p. 125). Using a three phase approach to transition including pre-transition, transition, and transfer, helps prepare the adolescent and address these challenges (Sable et al., 2011). The adolescent's family is a part of the transition process. During this time, parents are preparing for their child's independence as it relates to managing their medical condition (McManus et al., 2013; Sable et al., 2011). Transition education should begin between $12-14$ years of age and continue until ages $18-20$ whether or not a transfer of care takes place (American Academy of Pediatrics, American Academy of Family Physicians, \& American College of Physicians-American Society of Internal Medicine, 2002; Sable et al., 2011). Adolescents with CHD should receive education in core curriculum concepts described by Sable et al. and should be discussed over a minimum of two visits for older teens when starting later then the recommended age..

Providers need to be aware of the following risk factors that have been identified in specific CHD populations that are less likely to have adequate transition education: 
males, ethnicity other than non-Hispanic white, non-English speaking, $\leq 399 \%$ of federal poverty level, lack of a medical home, lack of insurance or public coverage (Lotstein et al., 2009; McManus et al., 2013, p. 1093). Age was not a statistically significant factor, however developmental or emotional delay or developmental readiness were important and could determine whether transition would even take place (Sable et al., 2011).

Another factor within the health care environment that makes transfer of care difficult for adolescents with CHD is the lack of trained medical providers for adults with CHD. It is essential that patients with CHD be followed by a provider that is trained and specializes in ACHD since it is very different from adult onset heart disease diagnoses (Sable et al., 2011). As of 2011, only 30\% of patients with ACHD were seen by a properly trained adult provider (Sable et al.). Anticipatory guidance related to sexual activity, contraception, and unintended pregnancy, which are standard topics of discussion by providers with adolescents and young adults, become much more complicated for adolescents with CHD and ACHD . Certain CHD diagnoses, such as hypoplastic left heart syndrome, make pregnancy very high risk for the patient. In addition, certain types of contraceptives can interact with the patient's medications or present other unintended health consequences (Said, Dearani, Silversides, Martinez, \& Drajpuch, 2011). Collaboration between providers skilled in adult management of CHD and obstetricians or gynecologists is needed to provide proper care and counseling for CHD patients; however currently there is often suboptimal care provided due to the lack of properly trained providers (Said et al., 2011).

The recommendation is that transition care would begin around age 13, and be included in or immediately following their scheduled cardiology provider visit. This type 
of solution is based on the definition of "purposeful, planned movement" since time is scheduled strictly for the purpose of educating and preparing the adolescent to selfmanage their health condition and for their parents to begin transferring the management to their child without fear or anxiety since both would receive education and support (American Academy of Pediatrics et al., 2002; Anthony et al., 2009; Jalkut \& Allen, 2009; Lotstein et al., 2009; Modi et al., 2012; Moons et al., 2009; Sable et al., 2011).

\section{Nursing Theory and Evidence Based Practice Model}

The EBPI San Diego Consortium Model - “The Eight A's” was chosen for this project (Figure 1) (Brown \& Ecoff, 2007). The model assesses the problem, asks a question, acquires evidence, appraises the evidence, applies a practice change, analyzes the change, advances the results and adopts the new change. This project is informed by Dorothea Orem’s Self-Care theory (Dorothea Orem's Self-Care Theory, 2012). Orem’s theory of self-care consists of three categories: 1) Universal self-care requisites; 2) Developmental self-care requisites and 3) Health deviation self-care requisites. The category of health deviation self-care requisites is directly related to the adolescents with CHD. Within this category the patient should be able to: 1) obtain proper medical assistance; 2) have knowledge of and address and clinical issues related to their condition; 3) carry out any prescribed treatments; 4) accept themselves and their particular health state and 5) learn to adapt to living with the effects and limitations of their condition (Dorothea Orem's Self-Care Theory, 2012).

\section{Tools Used}

\section{Knowledge Assessment}


Moons et al. (2001), developed a knowledge assessment tool known as the Leuven Knowledge Questionnaire for Congenital Heart Disease (LKQCHD). The LKQCHD is a validated tool that measures knowledge in four domains: 1) the defect or disease and treatment; 2) prevention of complications, including endocarditis; 3) physical activities; and 4) reproductive issues (Moons et al., 2001, p. 75). The LKQCHD was used to assess each participant's knowledge before receiving purposeful education, and then used to evaluate the change in knowledge following the educational interaction (Figure 2).

In a study done byVan Deyk et al. (2010), it was shown that a score on the LKQCHD of 50\% to $80 \%$ demonstrated moderate knowledge by the patient. A score of less than $50 \%$ on the LKQCHD demonstrated poor knowledge of their heart defect, what follow up visits were for and the symptoms that indicated a deterioration of their condition. Providing transition education that facilitated adolescents achieve a score of greater than $50 \%$ on the LKQCHD demonstrated that patients have the basic knowledge to begin self-managing their condition.

For this reason, it is important to evaluate if the participants achieved this outcome. The outcome evaluation question is, "What percentage of participants scored greater than $50 \%$ on the LKQCHD?” The indicator for this evaluation question is the LKQCHD scores. By answering this question, the post intervention score will show if the transition education provided was adequate to provide patients enough knowledge to score $50 \%$ or greater on the LKQCHD as shown by Van Deyk et al. (2010). Patient knowledge about the name of their heart defect, timing of follow up appointments and 
their medication regimen will also be evaluated on the LKQCHD. These are important factors in self-management.

\section{Adult Congenital Heart Association "Health Passport”}

The foundation of the teaching provided was through the use of an interactive “health passport” designed by the Adult Congenital Heart Association (ACHA) (Adult Congenital Heart Association, 2011). The AHA recommended that a "health passport” be used as an interactive tool within a resource binder (Sable et al., 2011). Elements of the education focused on clinical self-management and met the guidelines set by the AHA and AAP Consensus Statement. The passport provided opportunities for the patient to input specific details such as their contact information and who to contact in case of emergency. The patient also inputs their diagnosis, previous interventions, and medications, including dose and frequency. Information can be updated as needed. Patients also attached a print out of their current electrocardiogram (ECG) for baseline comparison. Completion of the interactive passport in a binder, along with handouts unique to their specific condition, provided a place for the patient to insert the copies of the medical records they receive and have everything in one place to help them selfmanage their medical needs.

\section{Methods}

The overall goal of this project is to develop and implement a pilot transition education program for adolescent with CHD to increase knowledge regarding their specific diagnosis and management as they enter adulthood.

\section{Design}


This was a quality improvement project that implemented an evidence-based pilot transition education program in an outpatient cardiology clinic setting using a pre and post-intervention design. The education provided was based on the American Heart Association (AHA) 2011 recommendations for managing transition for adolescents with CHD and the American Academy of Pediatrics (AAP) 2011 Clinical Report that supports health care transition.

\section{Setting}

The implementation of a pilot transition education program for adolescents with CHD took place in an outpatient pediatric cardiology clinic. The clinic is a hospital based clinic located in an urban area in the southwestern region of the United States. The cardiology clinic is an academic center which employees 16 pediatric cardiologists. Patients are traditionally seen in this clinic into adulthood, with a small percentage transferring care to an adult provider at age 21.

A needs assessment at the pediatric cardiology clinic related to transition demonstrated that no formalized adolescent transition program was in place. Transition education was limited and inconsistent between providers with time and scheduling being a large barrier identified. A further identified concern is that some of the patients do not transfer to an adult healthcare setting due to the lack of trained providers in CHD. The lack of providers trained to care for adults with CHD results in overload on the pediatric system. Many patients in their 20's are still be seen in the pediatric clinic, and are typically accompanied by their parents to the clinic appointments.

Another impetus for the implementation of this evidence-based project is that the hospital identified the need for a transition program in Cardiology in their 2014 operating 
goals. In addition, the Director for Cardiology made it a priority for the department to develop a formal standardized transition program for the adolescents with CHD.

\section{Participants}

A convenience sample of patients between the ages of 16 - 18 years old with a confirmed cardiac condition seen in an outpatient cardiology clinic during the project period of 4 months were identified for the pilot transition program. Patients were excluded if they had a diagnosis of developmental delay or were unable to read, write or speak English. Patients participated in the pilot transition program as an additional component of their scheduled routine follow up cardiac visit.

The Institutional Review Boards associated with the organization reviewed and granted exempt approval for this project. This project met criteria as an evidence-based quality improvement program.

\section{Data Collection}

Demographic data (Table 1) as well as clinical characteristics were collected such as age, gender, ethnicity, primary cardiac diagnosis, additional cardiac diagnoses, and complexity of cardiac diagnosis. Each set of pre and post completed LKQCHD were numbered 1-20 and de-identified of any patient information. The pre and post scores were input into an excel spreadsheet by number. An additional spreadsheet was created that looked at each individual question pre and post education.

\section{Implementation}

The "health passport" described above was printed on 8 1⁄1/2 x 11 paper and placed in a binder. Each binder was then individualized with additional handouts based on the patient's diagnosis and any procedures that may have been used as treatment. 
Informational handouts for any medications the patient is taking were also included. The binder was then used as a resource during the educational portion of the patient's visit.

On the day patients were scheduled for their cardiology appointment, many also had diagnostic testing such as: ECG, chest radiograph and in some instances echocardiography or exercise stress testing. At the time patients checked in, they were provided with the LKQCHD and explained that it was to assess their knowledge about their cardiac condition. They were encouraged to independently answer the questions without parental input to accurately assess their knowledge. The pre interaction questionnaire was then scored while they underwent their scheduled diagnostic testing.

After the diagnostic testing was completed and prior to their visit with the cardiologist, education was provided to the patient. The interactive "health passport" binder was used as the educational tool. The education was focused on the knowledge gaps demonstrated on the patient's completed LKQCHD. Each educational session took approximately 30 minutes. The education included the following elements: 1) cardiac defect or condition, and surgical or procedural interventions; 2) medications; 3) prevention of complications; 4) physical activity recommendations; 5) sexual health and genetic transmission; 6) for the female patients - contraception type and pregnancy risk. These topics are recommended by the AHA and are covered in the LKQCHD (Sable et al., 2011).

At the end of the session, the patient's completed the LKQCHD again to determine if their knowledge increased. They were also provided a health records request form and assisted with completion of the form, and instructed on the process to obtain 
their medical records to place in to their binder. The parents were also asked to complete a short informal survey to provide their feedback on the education provided.

\section{Results}

A total of 20 patients completed the pilot transition education program during the project period. The project participants were 60\% Hispanic, 30\% Caucasian and 10\% Pacific-Islander, with ages ranging from 16 to 18 years old. Patients were 60\% male and $40 \%$ female. Forty percent of patients had more than one cardiac diagnosis, and $70 \%$ had undergone either surgical or procedural intervention or both. The complexity of cardiac diagnosis were fairly evenly distributed: simple 30\%, moderate 35\% and complex 35\% (Table 1).

The mean score for the pre-intervention LKQCHD was 43.6\% (23.5\% - 78.8) and post-intervention LKQCHD was 89.4\% (58.1\% - 100\%). (Figure 3) This represents an increase in LKQCHD scores of over 200\%, with three patients scoring 100\% postintervention. (Table 2). The largest pre intervention knowledge gap in the overall group was related to identifying risk factors associated with endocarditis. Only $5 \%$ answered the multiple answer question correctly pre intervention. Post intervention, the knowledge score related to endocarditis rose to $80 \%$. Another large knowledge gap identified in female participants was related to appropriate contraception options and pregnancy risks. Of the eight females in the program, none of them were able to correctly answer what type of contraception was safe for them to use, and only one knew the risks associated with pregnancy and CHD. Post intervention knowledge scores for both contraception and pregnancy risk were $100 \%$. Overall, complexity of diagnosis did not have an impact on knowledge scores. 


\section{Discussion}

Providing structured transition education that is also individualized to the patient increases their knowledge about their cardiac condition. Providing specific handouts individualized to their CHD will provide a source of information as they assume more of the required management. Late adolescence is a time of transition and many of the patients in this project were preparing to leave for college. This intervention provided them with a binder including significant information about their CHD and medical records that can travel with them. Late adolescence can also be a time when many risk taking behaviors are on the rise. Studies have shown that most adolescents have their first sexual encounter by age 16 years and that $50 \%$ will result in pregnancy within six months of this encounter (Sable et al., 2011). Being a pregnant teenager has its own set of challenges, but for an adolescent with CHD it could have life threatening consequences. The finding that all of the female patients in this project lacked knowledge about what type of contraception was safe for them to use was not too surprising. During cardiology visits, the parent(s) is in the room and much of the interactive conversation is with them. Most of the conversation during the visits centers on how the patient is feeling clinically, what test results may have shown and if there are any upcoming surgeries or procedures. Cardiologists are under time constraints in the clinical environment and having a conversation about psych-social issues may seem like a luxury unless something is having a direct impact on the patient's clinical status. Adolescents are also reluctant to discuss issues such as sexual activity in front of their parents and may not realize that their condition has any relationship to contraception or how pregnancy could put them at high risk of complications including death. For all adolescent females, preventing 
unplanned pregnancy is important for many reasons, and for this population it could be a life or death issue. Contraception options containing estrogen are contra-indicated for females with certain cardiac diagnoses or taking anticoagulants (Sable et al., 2011). There are many progestin only options which include long acting reversible contraception choices such as implants or intra-uterine devices. In a structured transition program, these discussions could take place without taking time away from clinical topics that are discussed in the regular cardiology visit.

An informal satisfaction survey was given to parents at the end of the educational session. Parents completed this while their adolescent completed the post-intervention LKQCHD. It consisted of three questions with responses being on a five point Likert scale.

Some of the themes identified from comments on the survey were:

1) Education was appropriate for patients developmental level

2) Made patient feel respected

3) Parent felt adolescent better prepared for going away to college.

4) Parent felt adolescent more aware of the seriousness of their condition It is often thought that parents are not willing to hand off care to their adolescent. The comments on the survey demonstrated that parents are ready and willing to pass on responsibility to their adolescent when proper transition education takes place.

Pediatric nurse practitioners (PNP) and advanced practice pediatric nurses (APRN) are educated in adolescent chronic illness and health promotion, and have strong skills in patient education. Working as a team with the cardiologists and the parents, they are in a position to assess the adolescent for readiness, provide developmentally 
appropriate education, and assess the effectiveness and ultimate outcome of the education. The AHA and a number of additional sources support the role of PNP's and APRN's in the transition process (Betz, 2013; Goossens et al., 2014; Jalkut \& Allen, 2009; Moons et al., 2008; Reid et al., 2004; Sable et al., 2011).

\section{Limitations of the Project}

The time frame for the project was August through December. Due to the limited amount of time, patients could only receive an initial assessment of their knowledge via the LKQCHD. Patients could also only receive one educational session during this time frame. The recommendations from the AHA are to have a minimum of two educational sessions prior to age 18 . The decision was made to choose patients between the ages of 16 - 18 years old since they are closer to the age of independence even though the recommendation is to begin transition planning between ages 12-14 years old. Developmental delay was an exclusion criteria, however this is not a contraindication to transition education, and future programs would include interventions that are developmentally appropriate transition programs.

Another limitation is that assessment for readiness was not performed as part of this intervention. Ideally, patients would be assessed and begin transition based on their age and developmental readiness.

Although the cardiology group has multiple providers, all of the patients in this project were followed by one cardiologist since his patients typically were older adolescents. Differences may have been found if patients seeing other providers were included.

\section{Implications for Nurse Practitioner Clinical Practice}


For patients that transfer care to an adult healthcare environment, the NP may act as a liaison with the adult provider and still provide intermittent care during the transition-transfer process. This can help assure continuity of care and a smooth transition.

The process used for this project can be applied to other chronic health conditions to assist those adolescents in preparing to self-manage their specific health care condition. Long term outcomes could be followed once a formal transition program is in place (reduced ED visits, hospitalizations, compliance with regularly scheduled visits).

Developmentally appropriate transition education for adolescents with CHD can provide this patient population with the skills to self-manage their condition as they enter adulthood. This may help reduce healthcare costs due to "lost to follow up" and requiring emergency care as well as prolonged hospitalizations as a result of unmanaged clinical deterioration. The role of a NP as the "Transition Coordinator" can ensure developmentally appropriate education for adolescents with CHD as well as a continuity of care for ACHD as they begin navigating the adult healthcare system. NP's are in a unique position to act as a liaison between the pediatric and adult health care system while collaborating with providers to manage the patient's transition and transfer to appropriate care. 


\section{References}

Adult Congenital Heart Association (Producer). (2011). Personal Health Passport. Retrieved from http://www.achaheart.org/Portals/0/pdf/ACHAPassport.pdf

American Academy of Pediatrics, American Academy of Family Physicians, \& American College of Physicians-American Society of Internal Medicine. (2002). A consensus statement on health care transitions for young adults with special health care needs. Pediatrics, 110(Supplement 3), 1304-1306.

American Academy of Pediatrics, American Academy of Family Physicians, American College of Physicians, Transitions Clinical Report Authoring Group, Cooley, W. C., \& Sagerman, P. J. (2011). Supporting the health care transition from adolescence to adulthood in the medical home. Pediatrics, 128(1), 182-200. doi: 10.1542/peds.2011-0969

Anthony, S. J., Martin, K., Drabble, A., Seifert-Hansen, M., Dipchand, A. I., \& Kaufman, M. (2009). Perceptions of transitional care needs and experiences in pediatric heart transplant recipients. American Journal of Transplantation, 9, 614-619. doi: 10.1111/j.1600-6143.2008.02515.x

Betz, C. L. (2013). Health care transition for adolescents with special healthcare needs: where is nursing? Nursing outlook, 61(5), 258-265. doi: 10.1016/j.outlook.2012.08.009

Blum, R. W. M., Garell, D., Hodgman, C. H., Jorissen, T. W., Okinow, N. A., Orr, D. P., \& Slap, G. B. (1993). Transition from child-centered to adult health-care systems for adolescents with chronic conditions: A position paper of the Society for 
Adolescent Medicine. Journal of Adolescent Health, 14, 570-576. doi: http://dx.doi.org/10.1016/1054-139X(93)90143-D

Brown, C., \& Ecoff, L. (2007). Evidence-Based Practice Institute Model.

Department of Health and Human Services. (2013). Healthy People 2020: Topics and Objectives.

Dorothea Orem's Self-Care Theory. (2012). Dorothea Orem's Self-Care Theory. Retrieved December 1, 2013 http://currentnursing.com/nursing_theory/self_care_deficit_theory.html

Goossens, E., Van Deyk, K., Zupancic, N., Budts, W., \& Moons, P. (2014). Effectiveness of structured patient education on the knowledge level of adolescents and adults with congenital heart disease. European Journal of Cardiovascular Nursing, 13(1), 63-70. doi: 10.1177/1474515113479231

Jalkut, M. K., \& Allen, P. J. (2009). Transition from pediatric to adult health care for adolescents with congenital heart disease: a review of the literature and clinical implications. Pediatric Nursing, 35(6), 381-387.

Lotstein, D. S., Ghandour, R., Cash, A., McGuire, E., Strickland, B., \& Newacheck, P. (2009). Planning for health care transitions: results from the 2005-2006 national survey of children with special health care needs. Pediatrics, 123(1), e145-152. doi: 10.1542/peds.2008-1298

McManus, M. A., Pollack, L. R., Cooley, W. C., McAllister, J. W., Lotstein, D., Strickland, B., \& Mann, M. Y. (2013). Current status of transition preparation among youth with special needs in the United States. Pediatrics, 131, 1090-1097. doi: 10.1542/peds.2012-3050 
Modi, A. C., Pai, A. L., Hommel, K. A., Hood, K. K., Cortina, S., Hilliard, M. E., . . Drotar, D. (2012). Pediatric self-management: A framework for research, practice, and policy. Pediatrics, 129(2), e473-e485. doi: 10.1542/peds.2011-1635

Moons, P., De Volder, E., Budts, W., De Geest, S., Elen, J., Waeytens, K., \& Gewillig, M. (2001). What do adult patients with congenital heart disease know about their disease, treatment, and prevention of complications? A call for structured patient education. Heart, 86(1), 74-80. doi: 10.1136/heart.86.1.74

Moons, P., Hilderson, D., \& Van Deyk, K. (2008). Implementation of Transition Programs can Prevent Another Lost Generation of Patients with Congenital Heart Disease. European Journal of Cardiovascular Nursing, 7(4), 259-263. doi: 10.1016/j.ejcnurse.2008.10.001

Moons, P., Pinxten, S., Dedroog, D., Van Deyk, K., Gewillig, M., Hilderson, D., \& Budts, W. (2009). Expectations and experiences of adolescents with congenital heart disease on being transferred from pediatric cardiology to an adult congenital heart disease program. Journal of Adolescent Health, 44, 316-322. doi: 10.1016/j.jadohealth.2008.11.007

Reid, G. J., Irvine, M. J., McCrindle, B. W., Sananes, R., Ritvo, P. G., Siu, S. C., \& Webb, G. D. (2004). Prevalence and correlates of successful transfer from pediatric to adult health care among a cohort of young adults with complex congenital heart defects. Pediatrics, 113(3 Part 1), e197-205.

Rosen, D. S. (2004). Transition of young people with respiratory diseases to adult health care. Paediatric Respiratory Reviews, 5(2), 124-131. doi: http://dx.doi.org/10.1016/j.prrv.2004.01.008 
Sable, C., Foster, E., Uzark, K., Bjornsen, K., Canobbio, M. M., Connolly, H. M., .. . Williams, R. G. (2011). Best practices in managing transition to adulthood for adolescents with congenital heart disease: The transition process and medical and psychosocial issues: A scientific statement from the American Heart Association. Circulation, 123, 1454-1485. doi: 10.1161/CIR.0b013e3182107c56

Said, S. M., Dearani, J. A., Silversides, C. K., Martinez, R. M., \& Drajpuch, D. B. (2011). Longer-term issues for young adults with hypoplastic left heart syndrome: Contraception, pregnancy, transition, transfer, counselling, and re-operation. Cardiology in the Young, 21, 93-100. doi: 10.1017/S1047951111001661

U.S. Department of Health and Human Services, Health Resources and Services Administration, \& Bureau., M. a. C. H. (2013). The National Survey of Children with Special Health Care Needs Chartbook 2009-2010. from http://mchb.hrsa.gov/cshcn0910/state/pages/ca.html

Van Deyk, K., Pelgrims, E., Troost, E., Goossens, E., Budts, W., Gewillig, M., \& Moons, P. (2010). Adolescents' understanding of their congenital heart disease on transfer to adult-focused care. American Journal of Cardiology, 106, 1803-1807. doi: 10.1016/j.amjcard.2010.08.020 